\title{
Controversial Role of Gamma-Glutamyl Transferase Activity in Cisplatin Nephrotoxicity
}

\author{
Lukas Fliedl ${ }^{1}$, Matthias Wieser ${ }^{1}$, Gabriele Manhart ${ }^{1}$, Matthias P. Gerstl ${ }^{1}$, \\ Abdulhameed Khan ${ }^{2}$, Johannes Grillari ${ }^{2,3}$ and Regina Grillari-Voglauer ${ }^{1,2,3}$ \\ ${ }^{1}$ ACIB, Vienna, Austria; ${ }^{2}$ Department of Biotechnology, University of Natural Resources and Life Sciences Vienna, \\ Vienna, Austria; ${ }^{3}$ Evercyte, Vienna, Austria
}

\begin{abstract}
Summary
Nephrotoxicity of chemotherapeutics is a major hindrance in the treatment of various tumors. Therefore, test systems that reflect mechanisms of human kidney toxicity are necessary. Cell culture based systems have to be developed to reduce animal testing. Renal proximal tubular epithelial cells are of specific interest in this regard, as they reabsorb substances from human primary urine filtrates and thus are exposed to urinary excreted xenobiotics and are a major target of cisplatin toxicity. While animal studies using gamma glutamyl transferase (GGT) knock-out mice or GGT inhibitors show that GGT activity increases kidney toxicity of cisplatin, the use of various cell models gives contradictory results. We therefore used a cell panel of immortalized human renal proximal tubular epithelial (RPTECs) cell lines differing in GGT activity. Low GGT activity resulted in high cisplatin sensitivity, as observed in RPTEC-SV4O cells or after siRNA mediated knock-down of GGT in RPTEC/TERT1 cells that have high GGT activity. However, the addition of GGT did not rescue but also increased cisplatin sensitivity, and adding GGT inhibitor as well as substrate (glutathione) or product (cysteinyl-glycine) of GGT resulted in decreased sensitivity. While our data suggest that the use of cell panels is of value in toxicology and toxicogenomics, they also emphasize the complex interplay of toxins with the intracellular and extracellular microenvironment. We hypothesize that especially epithelial barrier formation and polarity of RPTECs need to be considered in toxicity models to validly predict the in vivo situation.
\end{abstract}

Keywords: GGT, nephrotoxicity, RPTEC/SV40, RPTEC/TERT1, HEPTEC/TERT

\section{Introduction}

Development of novel drugs is very cost intensive and time consuming. About one third fails due to toxicological concerns or lack of clinical safety (Kola and Landis, 2004; Jiang et al., 2007). Therefore, suitable model systems for reliable preclinical testing are of major importance to validly predict clinical safety and can reduce the costs of bringing new products to the market. The $3 \mathrm{R}$ principles to reduce animal testing were first formulated in 1959 (Russell and Burch, 1959) and efforts to this effect were intensified with the European Directive 86/609/EEC and its revision 2010/63/EU. Still the need for in vitro toxicity prediction systems remains urgent (Li, 2004; Dorato and Buckley, 2007). Apart from reducing the need for animal testing, such test systems can help us understand the mechanisms of drug toxicity and will support high throughput screening approaches
(Davila et al., 1998; Hartung, 2001; Dorato and Buckley, 2007; Astashkina et al., 2012).

Nephrotoxicity testing is of special interest as several compound groups, including aminoglycoside antibiotics, calcineurin inhibitors or platinum compounds, are known to be specifically toxic to the kidney. Further, an estimated $18-27 \%$ of acute kidney failures are caused by drugs (Loghman-Adham et al., 2012). This indicates that the test systems currently in use only cover certain aspects of nephrotoxic side effects. The major cell type that is hit by nephrotoxic substances is the renal proximal tubular epithelial cell (RPTEC) (Rush et al., 1984; Boogaard et al., 1990). These cells are involved in the blood clearance mechanism and resorption of essential metabolites (Smith et al., 2006) and are exposed to a high blood flow. Therefore, RPTECs seem to be the most appropriate cells for nephrotoxicity model systems (Li et al., 2006 and reviewed by Boogaard et al., 1990). 
So far, several RPTEC lines have been established from various organisms.

The well characterized porcine cell line LLC-PK1 (Hull et al., 1976) and rodent primary cells have been used to test cytotoxicity of chemicals and to predict nephrotoxicity. However, these models are limited by the fact that cells from different species may show different responses to the same drug and to other factors such as oxidative stress (Dietrich et al., 2001; Li, 2004; Parrinello et al., 2003), therefore the predictivity of animal cells for human cell responses is clearly limited.

Thus, human primary cells were assessed as model systems (Boogaard et al., 1990; Cummings et al., 2000; Li et al., 2006; Brown et al., 2008). Even though very valuable, these models are hampered due to limited population doublings, resulting in the need for large amounts of human tissue samples from which the cells must be prepared and therefore high variability in RPTEC cell behavior between isolations from different donors. Also, the human kidney cell line HK-2, immortalized by a recombinant retrovirus containing the human papilloma virus E6/E7 genes, has been used, but controversial results concerning this cell line's RPTEC-like characteristics, including drug transporter expression, have been published (Ryan et al., 1994; Wieser et al., 2008; Jenkinson et al., 2012). Moreover, for all cell lines used for toxicity testing so far alterations of the primary cell-like characteristics due to viral oncogenes used for immortalization were noted and are seen as major shortfalls (Stacey and Hartung, 2006), even though the use of conditional SV40 in combination with telomerase seems promising (Wilmer et al., 2010). Still, telomerase alone is regarded as the most appropriate strategy to immortalize cells without altering the primary cell-like key functionalities, and a large variety of different human cells have been successfully immortalized using hTERT (Bodnar et al., 1998; Chang et al., 2005). We have previously reported that introduction of hTERT alone is sufficient for immortalization and does not change the functional characteristics of epithelial cells of renal proximal tubules (Wieser et al., 2008). However, it may prove useful to employ a panel of cell lines originating from more than one individual to represent different genetic backgrounds and so improve predictivity.

In order to test the value of in vitro nephrotoxicity model systems, we have chosen cisplatin, a widely used chemotherapeutic agent against cancers of the testis, cervix, head, neck and lung (Kartalou and Essigmann, 2001; Townsend, 2003; Dos Santos et al., 2012). Moreover, its applicable dose is limited by an extremely narrow ratio of nephrotoxicity to therapeutic effect (Li et al., 2006). An important, but ambiguous, role in cisplatin toxicity has been attributed to GGT, the enzyme that catalyzes the first step of GSH degradation and produces cysteinylglycine (cysgly). GGT is essential for the recycling of cysteine from the extracellular space and provides the cell with cysteine required for de novo synthesis of intracellular GSH. However, even if in vivo models of GGT knockout mice and the use of GGT inhibitors in mice favor a role of GGT in toxification of cisplatin, results from in vitro models do not yet match the in vivo results. Specifically, some reports show toxic effects caused by metabolism of cisplatin through a pathway including GGT (Hanigan et al., 2001), whereas others see GGT as the main detoxification mechanism, where the direct GGT product cysgly or the GGT substrate glutathione (GSH) are covalently attached to cisplatin, rendering it nontoxic (Daubeuf et al., 2002, 2003; Paolicchi et al., 2003).

Here we report the characterization of a cell panel of RPTECs derived from different individuals and immortalized by different strategies in regard to their sensitivity to cisplatin. We show a surprising modulation of the cytotoxic effects of cisplatin by GGT, GSH, cysgly and OU749. We also show the effect of siRNA knockdown of the active GGT variant. Taken together, we suggest that GGT plays a complex role in cisplatin detoxification with interactions of the intra- and extracellular environment of cells playing a critical role. In addition, we propose that the novel human model cell lines tested here can be applied for studying GGT related nephrotoxic mechanisms as a cell panel, and propose that such cell panels might be useful for the prediction of nephrotoxicity and mechanisms of toxicity. However, limitations in classical two-dimensional cell culture became apparent from these results and suggest that cultivation conditions that mimic luminal and basolateral sides are necessary especially for polar epithelial cells.

\section{Materials and methods}

\section{Cell culture conditions}

All cell lines used within this study were cultivated at $37^{\circ} \mathrm{C}$ in a humidified atmosphere containing $7 \% \mathrm{CO}_{2}$. Confluent monolayers were passaged with a split ratio of 1:2-1:4 depending on the cell line. The cells were detached using $0.1 \%$ trypsin $/ 0.02 \%$ EDTA (Sigma), which was inactivated by addition of soybean trypsin inhibitor ( $1 \mathrm{mg} / \mathrm{ml}$, Sigma), followed by a centrifugation step (5 min, 170xg), and transfer of the resuspended cells to new culture vessels.

\section{Commercial cell lines}

RPTEC/TERT1 cells (Evercyte GmbH) and HEPTEC/TERT1/2 cells (Evercyte $\mathrm{GmbH}$ ) were cultivated in ProxUp and ProxUp-2 medium (Evercyte $\mathrm{GmbH}$ ), respectively. Prior to assays, cells were adapted to Optipro SFM ${ }^{\mathrm{TM}}$ (Invitrogen). HK-2 cells (ATCC) and RPTEC/SV40 cell lines were maintained in Optipro $\mathrm{SFM}^{\mathrm{TM}}$ (Invitrogen).

\section{Cell line establishment}

The study was approved by the local ethics commission (General Hospital of Vienna, Austria) and donors gave informed consent before providing urine samples. Thus, the study was performed in accordance with the Declaration of Helsinki.

Normal human renal proximal tubular epithelial cells (nRPTECs) and human exfoliated proximal tubular epithelial cells (HEPTECs) were isolated from urine samples as described in detail previously (Wieser et al., 2008, 2014) and cultivated in ProxUp-Pri medium (Evercyte $\mathrm{GmbH}$ ).

nRPTECs were transfected with a plasmid encoding SV40 early region (Voglauer et al., 2005) using Lipofectamin 2000 (Invitrogen) according to the manufacturer's instructions. Non- 
transfected cells were overgrown by SV40 early region expressing cells and these cells were further propagated as mass culture designated RPTEC-SV40 as described in detail previously (Fliedl et al., 2014).

For immortalization of HEPTECs, cells from 2 different donors were transduced with retroviruses encoding the catalytic subunit of human telomerase as described (Wieser et al., 2014). Twenty-four hours post transduction positively transduced cells were selected using $100 \mu \mathrm{g} / \mathrm{ml} \mathrm{G} 418$ (Invivogen) and arising clones were cultivated as mass culture designated HEPTEC/ TERT1 and HEPTEC/TERT2.

\section{GGT assay}

The protocol for determination of GGT activity was adapted from Meister et al. (1981). In brief, cells were cultured in 12 -well plates to full confluence and incubated with substrate (1 mM G-glutamyl-para-nitroanilide, $60 \mathrm{mM}$ Tris- $\mathrm{HCl} \mathrm{pH} 8.0$ and $20 \mathrm{mM}$ glycyl-glycine) for $20 \mathrm{~min}$ at RT. The enzymatic process was stopped by addition of $10 \%$ acetic acid (1:2). Para-nitroanilide release was determined with Tecan Infinite M200 at $405 \mathrm{~nm}$. Values were normalized to cell number as determined by Vicell. All tests were performed with biological and technical triplicates. For biological replicates, cells were grown and treated separately and technical replicates were three parallel measurements at the end of the protocol. While technical replicates were only used to evaluate stability of measurement, biological replicates were used to evaluate standard deviation. For supplementation experiments GGT (Sigma Aldrich; stock solution 10 units/ml in $0.2 \mathrm{M}$ 2-amino2-methyl-1,3-propandiol buffer pH 8.5) or OU749 (Cayman Chemical; stock solution $50 \mathrm{mM}$ in DMSO) were used in the concentrations indicated there.

Cytotoxicity assay

Cells were grown in 96-well plates. Before reaching confluence (approximately 90-95\%) they were incubated with the indicated concentrations of cisplatin (stock solution $3.33 \mathrm{mM}$ in DMSO) for $24 \mathrm{~h}$ followed by addition of $10 \mu \mathrm{MTT}(5 \mathrm{mg} / \mathrm{ml})$ per well for $4 \mathrm{~h}$. After solubilization of formazan by SDS in $0.1 \mathrm{M} \mathrm{HCl}$ results were measured with Tecan Infinite M200 at $570 \mathrm{~nm}$ and reference value at $690 \mathrm{~nm}$. For supplementation experiments cisplatin was coincubated with $1.5 \mathrm{mM}$ of cysteinylglycine (Sigma Aldrich; $6 \mathrm{mM}$ in PBS), $833 \mu \mathrm{M}$ GSH (Sigma Aldrich; $5 \mathrm{mM}$ in PBS), $1.66 \mathrm{U} / \mathrm{ml}$ GGT or $200 \mu \mathrm{M}$ OU749 for $2 \mathrm{~h}$ before addition to the cells. Vehicle controls were added accordingly in the respective assays. All tests were performed with biological triplicates, which were used to evaluate standard deviation. For calculation, values were standardized using the appropriate controls.

\section{GGT knockdown}

Cells were seeded $24 \mathrm{~h}$ prior to transfection with siRNA to reach approximately $50 \%$ confluence. For specific knock-down a pool of 4 pre-designed IBONI ${ }^{\circledR}$ siRNA (riboxx Life sciences) targeting GGT1 or GGT7 were used at a final concentration of $20 \mathrm{nM}$ as recommended by the manufacturer. Transfection was done according to the manufacturer's protocol using riboxxfect 100 (riboxx Life sciences). GGT assay and cytotoxicity assays were performed $48 \mathrm{~h}$ after transfection.

\section{RNA isolation, quantification and quality control}

Total RNA was isolated using Tri reagent (Sigma) according to the manufacturer's instructions. RNA concentration was determined by absorbance measurement on a Nanodrop ND-1000 spectrophotometer.

\section{QPCR}

For quantification of mRNA expression, $500 \mathrm{ng}$ of total RNA were reverse transcribed using the Dynamo cDNA synthesis kit (Fynnzymes). Quantitative real-time PCR was performed on a Rotor-Gene real-time cycler (Corbett Research) using 5x HOT FIREPol EvaGreen ${ }^{\circledR}$ qPCR Mix Plus (NO ROX) as indicated by the manufacturer. For quantification, serial dilutions of a standard were included in each run. Copy numbers were calculated after dynamic tube normalization and slope correction. Expression values were normalized to GAPDH and indicated as fold changes relative to control.

\section{Primers}

See Table 1

Tab. 1: Primers used in the study

\begin{tabular}{|l|l|l|l|}
\hline target & Accession number & forward & reverse \\
\hline ANPEP & NM_001150.2 & GTCCAGGGTCCAGGTTCCA & CTCCAACAGGCGAAGGTCAC \\
\hline CCBL1 & NM_004059.4 & TTGGGAACGAGGGGTGAAGC & TTCCACGGCAAAGTCTGGTG \\
\hline CCBL2 & NM_001008661.2 & CGCACCGGATAGCATGTTTTT & GGTCTGCAGCCAATTTGGTAA \\
\hline SCLC22A2 & NM_003058.3 & GACCGCATCGGACGCCGTTA & ACCAAGCCAAGCACGCCGAA \\
\hline GGT1 & NM_001032364.2 & CTGCTGGACGAGCCATGAAG & CACACAACAGGGCTGCAATG \\
\hline GGT7 & NM_178026.2 & CAATTACAGCGCCCTTGTGG & TGTTCTCGGGATACCAGGCT \\
\hline
\end{tabular}


Data analysis

All data were analyzed with GraphPad Prism. 4-parameter fit curves were used to calculate the IC50 values of cytotoxicity assays. Significance for comparison of $\mathrm{IC}_{50}$ values was calculated using t-tests. For evaluation of significance of GGT assays oneway ANOVA was used.

\section{Results}

\subsection{GGT determines cisplatin sensitivity in different immortalized human PTECs}

In order to test if different proximal tubular cell models (nRPTECs, HK-2, RPTEC/TERT1, RPTEC/SV40, HEPTEC/TERT1, HEPTEC/TERT2) respond differently to substances of known nephrotoxicity, we challenged them with cisplatin and measured the resulting cytotoxicity using the MTT assay (Mosmann, 1983; Daubeuf et al., 2003). The IC 50 was between 200 and 450 $\mu \mathrm{M}$ in all cell lines except for one: RPTEC/SV40 had a lower IC $_{50}$ value of $80 \mu \mathrm{M}$, demonstrating a higher sensitivity to cisplatin (Fig. 1).

Since GGT has been reported as a key enzyme in the cisplatin metabolizing mechanism, we analyzed GGT activity in all cell lines. Indeed, we observed a difference in GGT activity between RPTEC/SV40 and the other cell lines: While all other cell lines produced para-nitroanilide (pNA) levels of
0.25-0.56 $\mathrm{nmol} / \mathrm{min} / 10^{5}$ cells, the RPTEC/SV40 cell line only reached $0.03 \mathrm{nmol} / \mathrm{min} / 10^{5}$ cells (Fig. 2).

In order to test whether cells are sensitized to cisplatin by reduction of GGT, we performed siRNA knock-down of GGT in RPTEC/TERT1 cells. Expression of GGT variants was analyzed by conventional PCR in the cell panel as well as in kidney tissue (data not shown). siRNAs against the expressed GGT variants (GGT1 and GGT7) were used for specific knock-down. In GGT assays, the effects of untransfected control-siRNA, GGT1-siRNA and GGT7-siRNA were compared $48 \mathrm{~h}$ after transfection of RPTEC-TERT1. Negative control and GGT7 showed no effect, whereas GGT activity was decreased by $37 \%$ by siRNAGGT1 (Fig. 3A). When cisplatin was added to siRNA-GGT1 transfected cells, the sensitivity to cisplatin was increased, as was expected from the results with the low GGT active RPTEC/ SV40 cell line. The $37 \%$ reduction in GGT by siRNAs also led to an around $40 \%$ decrease in the $\mathrm{IC}_{50}$ (Fig. 3B).

\subsection{GGT in the extracellular space increases cisplatin sensitivity}

After demonstrating that knock-down of GGT increased cisplatin sensitivity, we expected that more GGT activity would decrease the sensitivity. GGT is a membrane bound enzyme with extracellular activity. We therefore supplemented cultures of the low GGT/highly sensitive RPTEC/SV40 cell line with purified GGT. GGT activity was observed after its addition to the supernatants

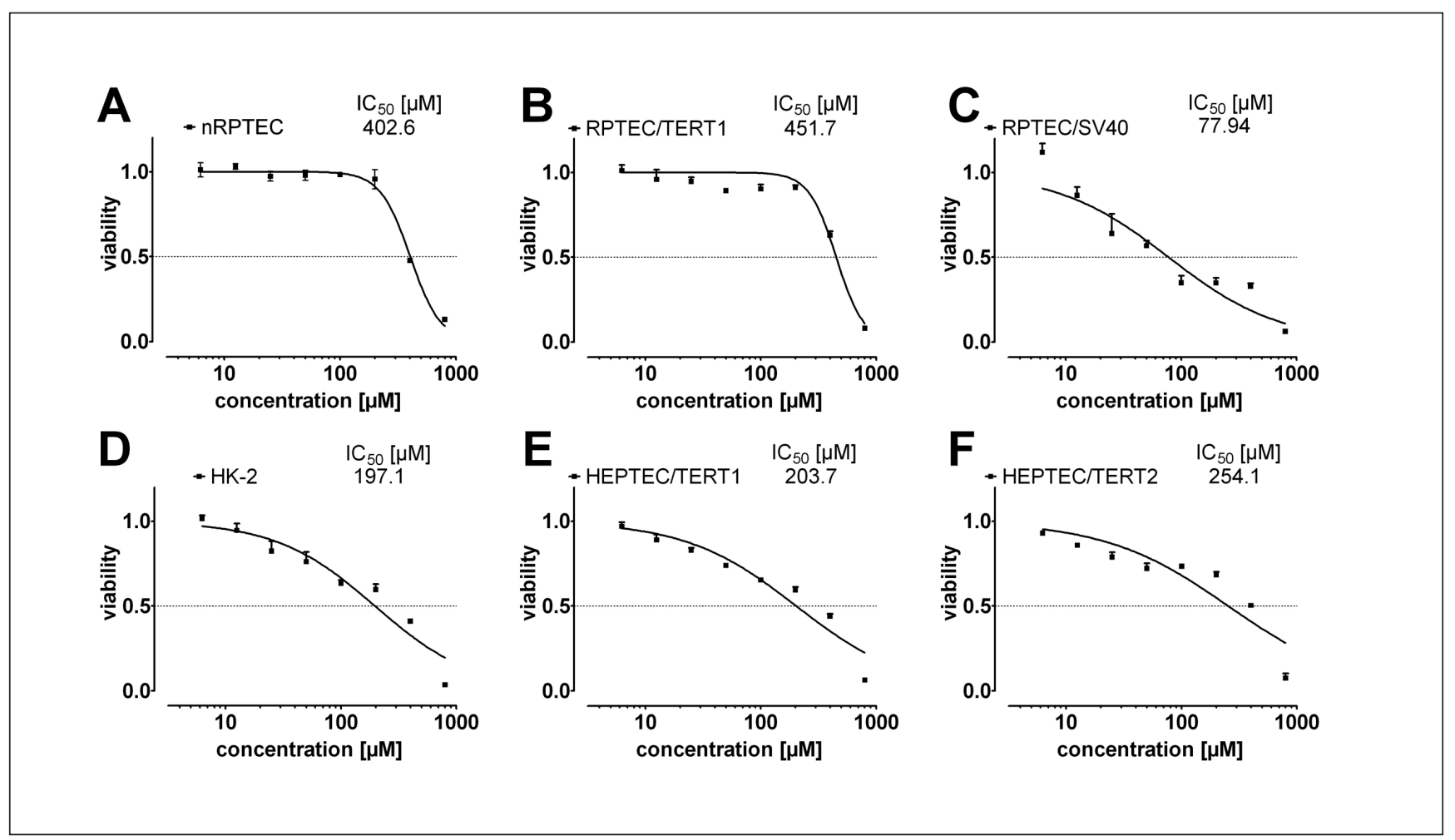

Fig. 1: Viability curves of human renal cell lines treated with different concentrations of cisplatin

Viability curves of human renal cell lines treated with different concentrations of cisplatin were determined by MTT assay and the $\mathrm{IC}_{50}$ values were calculated as 4 parameter fit curves. (A) nRPTECs (B) RPTEC/TERT1 (C) RPTEC/SV40 (D) HK-2 (E) HEPTEC/TERT1 (F) HEPTEC/TERT2. Biological triplicates were used to evaluate standard deviation. 


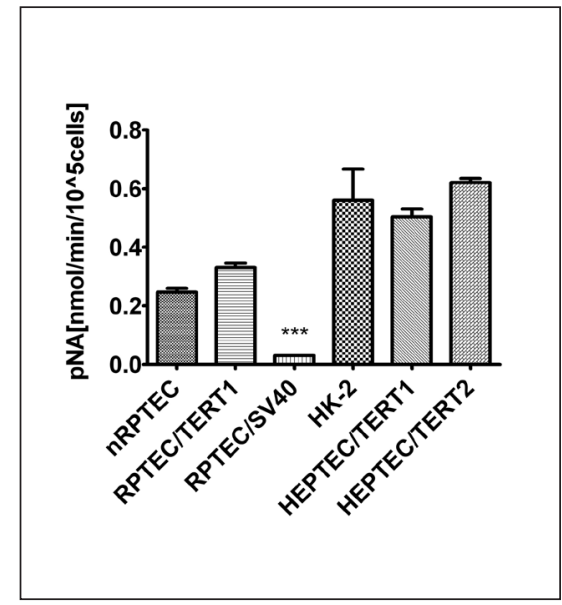

Fig. 2: GGT activity of the human renal cell line panel

GGT activity of all human renal cell lines in our panel. Biological triplicates were used to evaluate standard deviation. ${ }^{* \star *}, \mathrm{p}<0.05$ vs. other cell lines.

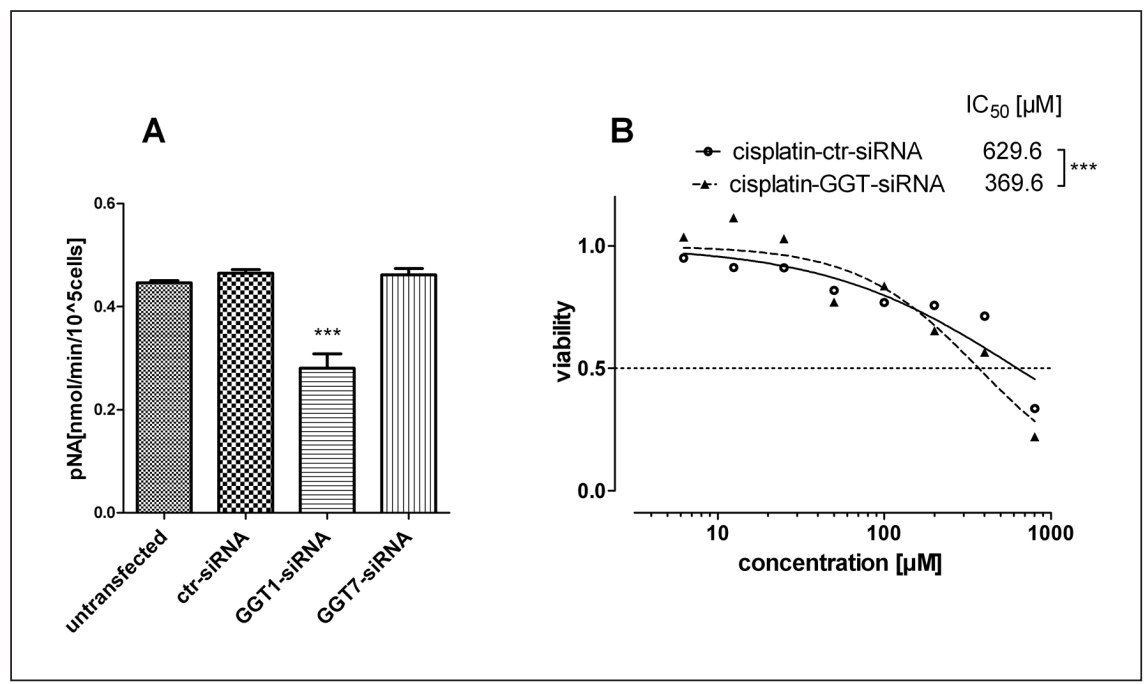

Fig. 3: Effects of siRNA treatment on GGT activity and cisplatin toxicity to RPTEC-TERT1

(A) GGT assay after siRNA transfection of RPTEC-TERT1. ${ }^{* * *}, \mathrm{p}<0.05$ vs. ctr-siRNA;

(B) effect of GGT1-siRNA on cisplatin toxicity on RPTEC-TERT1. ${ }^{* * *}, \mathrm{p}<0.05$.
A

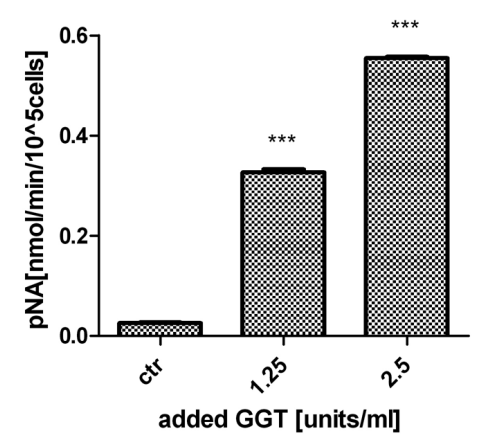

B

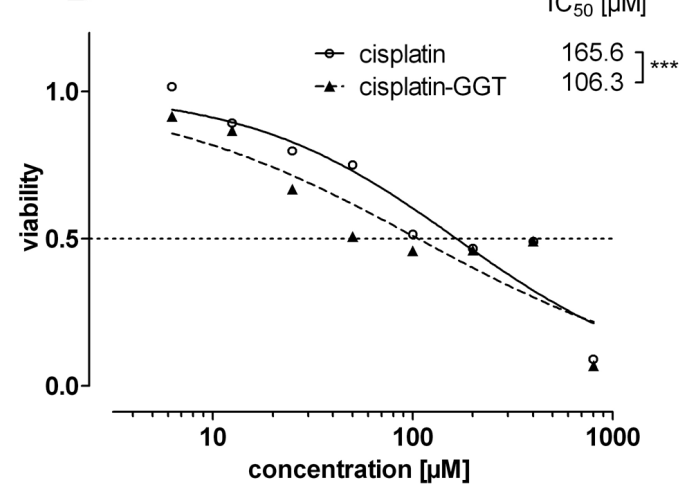

Fig. 4: Influence of GGT enzyme on GGT activity and cisplatin toxicity to RPTEC-SV40

(A) GGT assay showing the increased GGT activity caused by addition of the indicated units of the enzyme to RPTEC-SV40.

***, $p<0.05$ vs. ctr. (B) MTT assay of cisplatin treated cells after pre-incubation of RPTEC-SV40 with 1.66 units $/ \mathrm{ml} \mathrm{GGT.}$

Biological triplicates were used to evaluate standard deviation. ${ }^{* * *}, p<0.05$.

in a dose dependent way (Fig. 4A). When RPTEC-SV40 were incubated with 1.66 units/ml of GGT it resulted in higher activity, similar to RPTEC/TERT1. Surprisingly, however, GGT addition did not rescue from cisplatin sensitivity, but rather slightly increased it by decreasing the $\mathrm{IC}_{50}$ by about $35 \%$ (Fig. 4B).

In order to test if GGT inhibition would decrease sensitivity of the cells, we used the recently described specific GGT inhibitor OU749 (King et al., 2009). The GGT assay showed a dose dependent inhibition of GGT activity by OU749 using either RPTEC-SV40 (Fig. 5A) or RPTEC-TERT1 (Fig. 5C). Preincubation of cells before cisplatin treatment indeed rescued from cisplatin sensitivity by more than 200-fold in RPTEC/SV40
(Fig. 5B), i.e., the IC50 of RPTEC/SV40 cells was increased from 51.2 to around 10,000; even more striking RPTEC/TERT1 cells were completely rescued from cisplatin toxicity over the time period tested (Fig. 5D).

In order to test if substrate or product of GGT would have corresponding effects to overexpression or inhibition of GGT, we tested addition of GSH or cysgly before addition of cisplatin. When GSH was used for pre-incubation before cisplatin addition, a markedly decreased sensitivity of the cell line by more than 3.5 fold was observed (Fig. 6A). This protective effect is in line with published data. A consequence of an unbalanced high GGT activity in the extracellular space might be deple- 

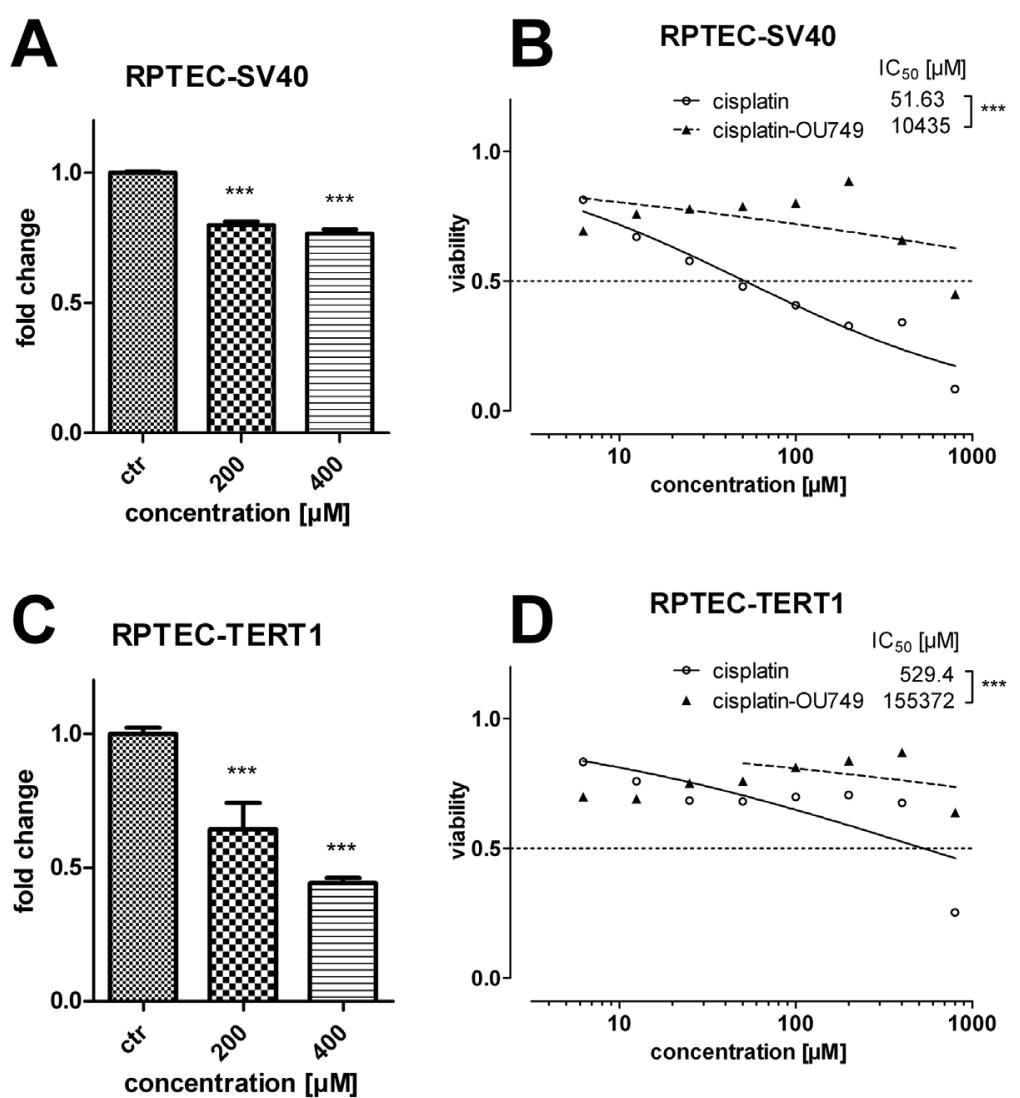

Fig. 5: Effect of GGT inhibitor OU749 on GGT activity and cisplatin toxicity of RPTEC-SV40 and RPTEC-TERT1 Fold change of GGT activity of RPTEC-SV40(A) and RPTEC-TERT1(C) treated with the indicated concentrations of OU749. ***, $\mathrm{p}<0.05$ vs ctr. Effect of $200 \mu \mathrm{M}$ OU749 on cisplatin toxicity in RPTEC-SV40(B) and RPTEC-TERT1(D). Biological triplicates were used to evaluate standard deviation. ${ }^{* * *}, \mathrm{p}<0.05$.

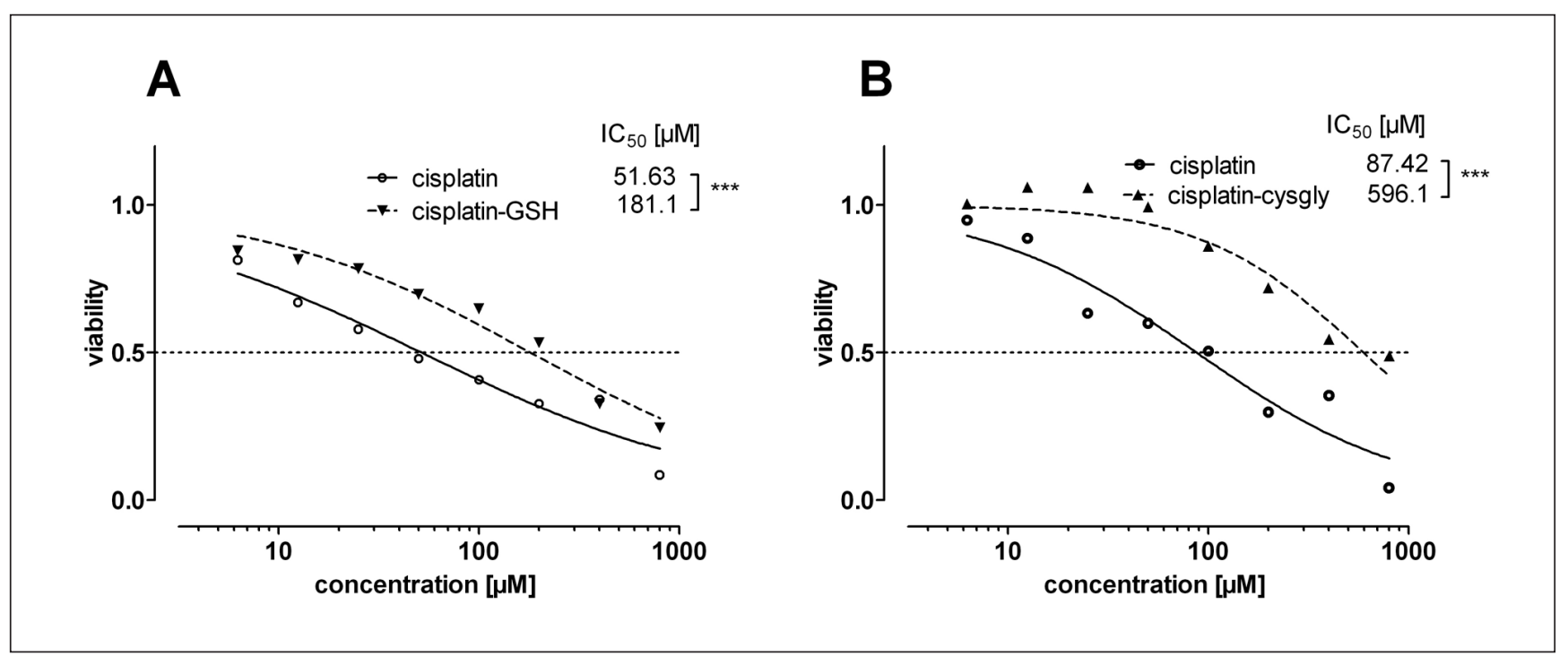

Fig. 6: Influence of substrate and product of GGT on cisplatin toxicity

MTT assay of cisplatin treated RPTEC-SV40 cells after pre-incubation with (A) $833 \mu \mathrm{M}$ glutathione (GSH) and (B) $1.5 \mathrm{mM}$ cysteinylglycine (cysgly). Biological triplicates were used to evaluate standard deviation. ${ }^{* *}, \mathrm{p}<0.05$. 
tion of GSH resulting in increased sensitivity. Pre-incubation of the cells with cysgly resulted again in a decreased cisplatin sensitivity and a more than 6-fold increase in IC 50 from 87.42 to 596.1 (Fig. 6B). Thus, blocking of GGT would also block cysgly formation and thus contribute to cisplatin sensitivity. These data would suggest that both GSH and the GGT product cysgly are capable of detoxifying cisplatin, while "unbalanced" addition of GGT to the supernatants might deplete GSH by its activity, but without supplying sufficient cysgly that might be further processed.

Furthermore, we tested mRNA levels of the following proteins possibly involved in the toxicity of cisplatin by qPCR: aminopeptidase N (ANPEP) is expressed in various cell types including the renal tubuli, cleaving peptides to enable cellular uptake; cystein-conjugate beta-lyase (CCBL) is an intracellular enzyme which metabolizes cysteine conjugates of certain halogenated alkenes and alkanes which may lead to formation of reactive metabolites causing nephron- and neurotoxicity and occurs in two variants; organic cation transporters OCT-2 (SLC22A2) in the kidney and other organs are responsible for excretion of many organic cations, drugs and environmental toxins. No correlation was observed between the mRNA expression of these proteins and the different sensitivity to cisplatin noted for the cell panel (Fig. S1 in supplementary file on http:// www.altex-edition.org).

Finally we evaluated the possible influence of growth rate on the cytotoxicity assays used. Growth rates were calculated from at least 6 serial passages. No significant differences in growth rates were noticed (Fig. S2 in supplementary file on http://www. altex-edition.org).

\section{Discussion}

Nephrotoxicity of cisplatin is a clinically important problem during chemotherapy. Toxicity to renal proximal tubular epithelial cells is a major factor in cisplatin nephrotoxicity and an enzyme specific for this cell type, GGT, has been discussed as a key enzyme in nephrotoxicity. Similarly, GGT seems involved in increasing resistance of tumors to cisplatin treatment.

However, reported results are controversial, claiming GGT activity either to enhance or to decrease cisplatin sensitivity of the kidney and/or RPTECs. We used a renal cell panel to test the potential of the cell lines as predictors of nephrotoxicity in order to elucidate the role of GGT. However, our experiments also result in controversial data, emphasizing that the simple question whether GGT enhances or decreases cisplatin toxicity in human RPTECs has a complex answer.

We here observed that one of the cell lines used, RPTECSV40, showed an increased sensitivity to cisplatin. This increased sensitivity was paired with a reduced GGT activity, which was also unique for RPTEC-SV40 compared to other cell lines. In keeping with this, the high GGT active RPTEC/ TERT1 cell line became more sensitive after siRNA mediated knock-down of GGT. The cell culture media was then complemented with the product of GGT, cysgly, which also rescued from increased sensitivity. These data would suggest that GGT is a "good" activity involved in detoxifiying cisplatin, and are consistent with previously published proposed mechanisms of GGT-dependent resistance to cisplatin (Daubeuf et al., 2002, 2003; Paolicchi et al., 2003). These proposed mechanisms were all based on findings using 2D cell cultures with cell lines that had varying degrees of similarity with RPTECs down to overexpression of GGT in HeLa cells. These findings also correlate with studies of different tumor and tumorigenic cell lines that have an increased GGT activity and a reduced sensitivity to cisplatin (Godwin et al., 1992; Fan et al., 1994).

In our attempts to more carefully study the mechanistic influence of GGT on proximal tubular epithelial cells, however, we also performed experiments adding purified GGT to the cell supernatants, and inhibiting GGT activity by addition of a specific inhibitor, OU749. Surprisingly, and in contrast to the siRNA experiment described above, GGT addition increased the sensitivity to cisplatin, and its inhibition decreased it, suggesting that GGT is the "bad" activity that activates and enhances cisplatin nephrotoxicity. Again, other recently published work also supports such an interpretation: A very strong indication that GGT is a damaging agent during cisplatin treatment came from GGT knock-out mice, where kidney failure and mortality after cisplatin treatment were observed at lower cisplatin doses (Hanigan et al., 2001), similar to when acivicin was employed as a GGT inhibitor (Hanigan et al., 1994). These results led Hanigan and co-workers to the conclusion that cisplatin is successively metabolized by GGT, dipeptidase and finally cystein-conjugate beta-lyase, finally forming an active thiol that causes nephrotoxicity (Townsend, 2003; Zhang and Hanigan, 2003). Paolicchi et al. (2003) reinterpreted these findings, as it had already been shown that systemic inhibition of GGT by acivicin lead to increased plasmatic levels of GSH in mice (Griffith and Meister, 1979). This finding was corroborated in GGT knockout mice (Lieberman et al., 1996). Therefore, an alternative explanation for the protective effect in GGT knockout or inhibited mice can be the plasmatic accumulation of GSH, which can offer direct protection (Paolicchi et al., 2003), and is also visible in our model system where addition of GSH is protective. A question that remains to be answered in this scenario would be whether the cisplatin is detoxified in the plasma before it can kill the tumor cells. In addition, an increase in cytotoxicity by addition of exogenous GGT might also come from depletion of GSH by GGT, as addition of GSH indeed also decreases sensitivity to cisplatin in our model systems, similar to the previous proposed models (Daubeuf et al., 2003; Paolicchi et al., 2003 and commentary of Pompella et al., 2006).

Thus, the paradoxical result of increase in cisplatin toxicity when purified GGT is exogenously added into the supernatant of RPTEC cells, could also be a result of increased oxidative burden caused by GGT (Dominici et al., 2005), even though it was published that overexpression of GGT and consequently higher activity can offer resistance to oxidative stress. Since the increased GGT expression was associated with higher expression levels of catalase (Giommarelli et al., 2008), the higher resistance of intracellular GGT overexpression might be indi- 


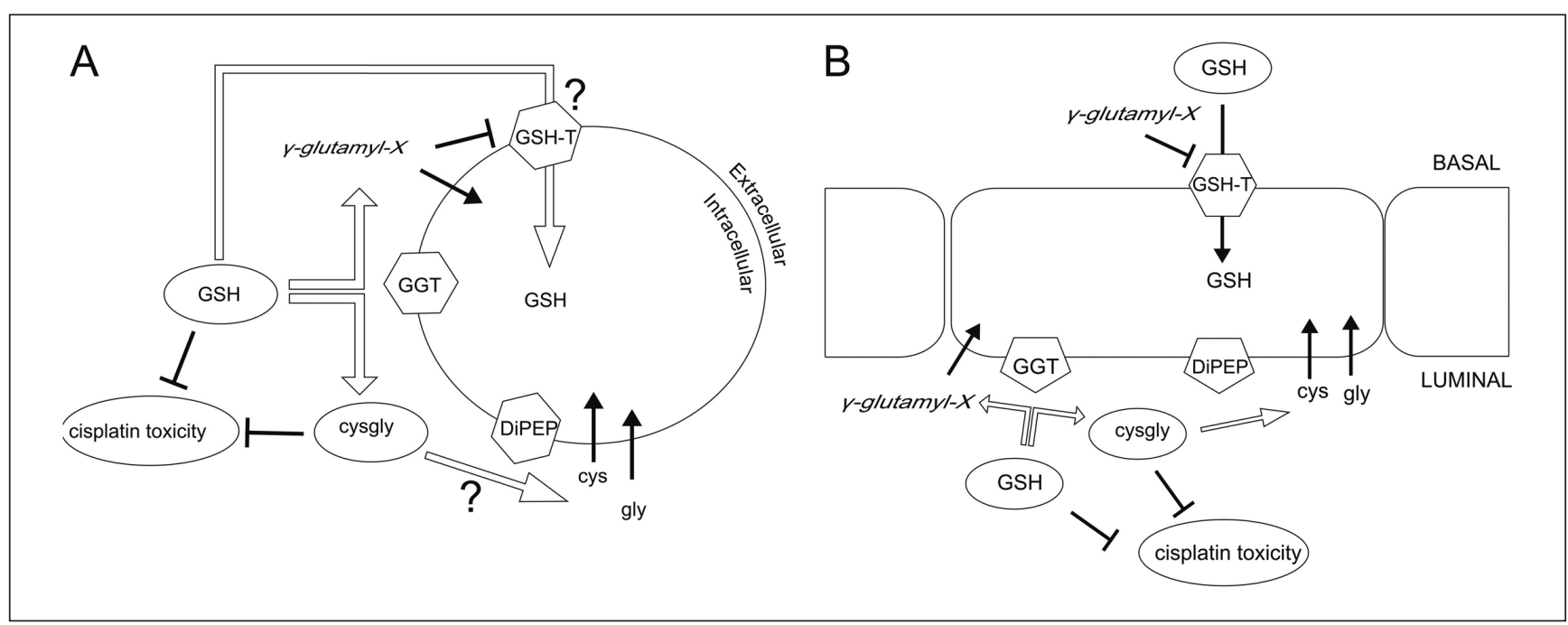

Fig. 7: Models for mechanism of cisplatin toxicity

(A) Model for 2D cell culture, similar to the previously proposed models, but now including a transporter for intact GSH (GSH-T) that is inhibited by a product of GGT, $\gamma$-glutamyl-X. All enzymes are in close proximity to each other and not in separate compartments. (B) In vivo 3D model: RPTECs are polar and form tight layers and in contrast to the 2D model GSH-T is located on the basolateral side, while GGT and the dipeptidase are on the luminal side. The different spatial organization as represented in the two models explains why contradictory results can be obtained depending on the model system used. Glutathione (GSH), $\gamma$-glutamyltransferase (GGT), cysteinylglycine (cysgly), DiPEP (dipeptidases)

rectly due to catalase activity and oxidative stress resistance. With addition of exogenous GGT the catalase expression would be unchanged and therefore our results might also be interpreted as a consequence of increased oxidative stress, in line with the results of Giommarelli et al. (2008).

How can such controversial data be explained and can GGT now be pinned down as "good" (helping to detoxify) or "bad" (increasing sensitivity of RPTECs) for kidney cells?

The cell culture models used so far all were performed in classical 2D cell culture. If we include data showing that intact GSH is transported into cells by the specific transporter GSH-T (Lash and Jones, 1983; Lash et al., 2007), which is inhibited by $\gamma$-glutamyl-X, a product of GGT (Lash and Jones, 1984; Hagen et al., 1988), we might suggest a process as outlined in Figure 7A. Thus, in RPTECs with low GGT activity (by siRNA or after SV40 immortalization), the extracellular GSH present in the medium might be depleted from the extracellular space by transport into the cell by the GSH-T transporter. In contrast, in the RPTECs with higher GGT activity, the GSH is converted to cysgly and $\gamma$-glutamyl-X. The $\gamma$-glutamyl-X in turn inhibits cellular uptake of intact GSH and thus might keep sufficient GSH in the extracellular space to decrease cisplatin sensitivity.

If however, GGT is added extracellularly, GSH is depleted quickly and efficiently to cysgly, which is further cleaved to the single amino acids, which are taken up. This cysgly cleavage seems to be very fast, so that overall less GSH and cysgly are available for detoxification. Thus the paradoxical situation arises that either reduced expression of GGT or addition of recombinant GGT both decrease sensitivity.

Why now, do we see lower cisplatin sensitivity when we add a specific GGT inhibitor? The answer might lie in the experimental set-up: GGT activity is blocked after changing the medium, so that already $\gamma$-glutamyl-X has been formed, blocking the GSH-T mediated uptake and thus extracellular depletion of GSH.

In vivo, however (Fig. 7B), RPTECs are polar and form tight layers with GSH-T on the basolateral side (Lash and Jones, 1983 and reviewed in Lash, 2010), while GGT and the dipeptidase are on the luminal side. Thus, lacking GGT activity (as in ko mice or using acivicin) reduces sensitivity as GSH levels are not depleted from the extracellular environment, because GSH$\mathrm{T}$ is spatially not available - in contrast to working with $2 \mathrm{D}$ cell culture models. It is clear that more detailed studies are necessary to support our model and to reconcile the controversial data. Moreover accumulation of GSH, oxidative stress by GGT activity and reduction of the latter by catalase could also play key roles in the nephrotoxicity of cisplatin.

In any case, we strongly suggest that such studies are undertaken using tight epithelial monolayers as "3-dimensional" models of RPTECs in transwell assays as used, e.g., by Brown et al. (2008). The spatial distribution of transport proteins in polar cells needs to be taken into account in future studies and might explain some differences in cisplatin resistance of cancer cells against cisplatin toxicity of RPTECs.

We here have included immortalized HEPTECs that can be derived non-invasively from any person and due to immortalization with hTERT are standardizable and retain their main RPTEC characteristics over many population doublings, similar to our RPTEC/TERT1 cell line (Wieser et al., 2008, 2014). These cells might turn out to be useful for generating cell panels of donors with different genetic backgrounds, diseases or treatments in the context of toxicogenomics and personalized medicine in general. 


\section{Conflict of interest}

RG and JG are cofounders of Evercyte $\mathrm{GmbH}$ and declare conflict of interest.

\section{References}

Astashkina, A., Mann, B. and Grainger, D. W. (2012). A critical evaluation of in vitro cell culture models for high-throughput drug screening and toxicity. Pharmacol Ther 134, 82-106. http://dx.doi.org/10.1016/j.pharmthera.2012.01.001

Bodnar, A. G., Ouellette, M., Frolkis, M. et al. (1998). Extension of life-span by introduction of telomerase into normal human cells. Science 279, 349-352. http://dx.doi.org/10.1126/ science.279.5349.349

Boogaard, P. J., Nagelkerke, J. F. and Mulder, G. J. (1990). Renal proximal tubular cells in suspension or in primary culture as in vitro models to study nephrotoxicity. Chem Biol Interac 76, 251-291. http://dx.doi.org/10.1016/0009-2797(90)90096-6

Brown, C. D. A., Sayer, R., Windass, A. S. et al. (2008). Characterisation of human tubular cell monolayers as a model of proximal tubular xenobiotic handling. Toxicol Appl Pharmacol 233, 428-438. http://dx.doi.org/10.1016/j.taap.2008.09.018

Chang, M. W.-F., Grillari, J., Mayrhofer, C. et al. (2005). Comparison of early passage, senescent and hTERT immortalized endothelial cells. Exp Cell Res 309, 121-136. http://dx.doi. org/10.1016/j.yexcr.2005.05.002

Cummings, B. S., Zangar, R. C., Novak, R. F. et al. (2000). Cytotoxicity of trichloroethylene and S-(1,2-dichlorovinyl)-L-cysteine in primary cultures of rat renal proximal tubular and distal tubular cells. Toxicology 150, 83-98. http://dx.doi.org/10.1016/ S0300-483X(00)00252-3

Daubeuf, S., Leroy, P., Paolicchi, A. et al. (2002). Enhanced resistance of HeLa cells to cisplatin by overexpression of gammaglutamyltransferase. Biochem Pharmacol 64, 207-216. http:// dx.doi.org/10.1016/S0006-2952(02)01118-8

Daubeuf, S., Balin, D., Leroy, P. et al. (2003). Different mechanisms for $\gamma$-glutamyltransferase-dependent resistance to carboplatin and cisplatin. Biochem Pharmacol 66, 595-604. http:// dx.doi.org/10.1016/S0006-2952(03)00343-5

Davila, J. C., Rodriguez, R. J., Melchert, R. B. et al. (1998). Predictive value of in vitro model systems in toxicology. Annu Rev Pharmacol Toxicol 38, 63-96. http://dx.doi.org/10.1146/ annurev.pharmtox.38.1.63

Dietrich, D.R., O'Brien,E., Stack, M.E. et al. (2001). Species- and sex-specific renal cytotoxicity of ochratoxin A and B in vitro. Exp Toxicol Pathol 53, 215-225. http://dx.doi.org/10.1078/09402993-00184

Dominici, S., Paolicchi, A., Corti, A. et al. (2005). Prooxidant reactions promoted by soluble and cell-bound gamma-glutamyltransferase activity. Methods Enzymol 401, 484-501. http:// dx.doi.org/10.1016/S0076-6879(05)01029-3

Dorato, M. A. and Buckley, L. A. (2007). Toxicology testing in drug discovery and development. Curr Protoc Toxicol 31, 19.1.1-19.1.35.

Dos Santos, N., Carvalho Rodrigues, M. A., Martins, N. M. et al. (2012). Cisplatin-induced nephrotoxicity and targets of nephroprotection: an update. Arch Toxicol 86, 1233-1250. http://dx.doi. org/10.1007/s00204-012-0821-7

Fan, S., El-deiry, W. S., Bae, I. et al. (1994). p53 gene mutations are associated with decreased sensitivity of human lymphoma cells to DNA damaging agents. Cancer Res 54, 5824-5830.

Fliedl, L., Manhart, G., Kast, F. et al. (2014). Novel human renal proximal tubular cell line for the production of complex proteins. J Biotechnol 176, 29-39. http://dx.doi.org/10.1016/j. jbiotec.2014.02.001

Giommarelli, C., Corti, A., Supino, R. et al. (2008). Cellular response to oxidative stress and ascorbic acid in melanoma cells overexpressing gamma-glutamyltransferase. Eur J Cancer 44, 750-759. http://dx.doi.org/10.1016/j.ejca.2008.02.010

Godwin, A. K., Meister, A., O’Dwyer, P. J. et al. (1992). High resistance to cisplatin in human ovarian cancer cell lines is associated with marked increase of glutathione synthesis. Proc Natl Acad Sci U S A 89, 3070-3074. http://dx.doi.org/10.1073/ pnas.89.7.3070

Griffith, O. W. and Meister, A. (1979). Glutathione: interorgan translocation, turnover, and metabolism. Proc Natl Acad Sci U S A 76, 5606-5610. http://dx.doi.org/10.1073/pnas.76.11. 5606

Hagen, T. M., Aw, T. Y. and Jones, D. P. (1988). Glutathione uptake and protection against oxidative injury in isolated kidney cells. Kidney Int 34, 74-81. http://dx.doi.org/10.1038/ki.1988.147

Hanigan, M. H., Gallagher, B. C., Taylor, P. T. et al. (1994). Inhibition of $\gamma$-glutamyl transpeptidase activity by acivicin in vivo protects the kidney from cisplatin-induced toxicity. Cancer Res 54, 5925-5929.

Hanigan, M. H., Lykissa, E. D., Townsend, D. M. et al. (2001). Gamma-glutamyl transpeptidase-deficient mice are resistant to the nephrotoxic effects of cisplatin. Am J Pathol 159, 18891894. http://dx.doi.org/10.1016/S0002-9440(10)63035-0

Hartung, T. (2001). Three Rs potential in the development and quality control of pharmaceuticals. ALTEX 18, Suppl 1, 3-13.

Hull, R. N., Cherry, W. R. and Weaver, W. (1976). The origin and charcteristics of a pig kidney cell strain, LLC-PK1. In Vitro 12, 670-677. http://dx.doi.org/10.1007/BF02797469

Jenkinson, S. E., Chung, G. W., van Loon, E. et al. (2012). The limitations of renal epithelial cell line HK-2 as a model of drug transporter expression and function in the proximal tubule. Pflugers Arch 464, 601-611. http://dx.doi.org/10.1007/s00424012-1163-2

Jiang, Y., Gerhold, D. L., Holder, D. J. et al. (2007). Diagnosis of drug-induced renal tubular toxicity using global gene expression profiles. J Transl Med 5, 47. http://dx.doi.org/10.1186/14795876-5-47

Kartalou, M. and Essigmann, J. M. (2001). Mechanisms of resistance to cisplatin. Mutat Res 478, 23-43. http://dx.doi. org/10.1016/S0027-5107(01)00141-5

King, J. B., West, M. B., Cook, P. F. et al. (2009). A novel, speciesspecific class of uncompetitive inhibitors of gamma-glutamyl transpeptidase. J Biol Chem 284, 9059-9065. http://dx.doi. org/10.1074/jbc.M809608200

Kola, I. and Landis, J. (2004). Can the pharmaceutical industry reduce attrition rates? Nat Rev Drug Discov 3, 711-715. http:// www.ncbi.nlm.nih.gov/pubmed/15286737

Lash, L. H. and Jones, D. P. (1983). Transport of glutathione 
by renal basal-lateral membrane vesicles. Biochem Biophys Res Commun 112, 55-60. http://dx.doi.org/10.1016/0006291X(83)91796-5

Lash, L. H. and Jones, D. P. (1984). Renal glutathione transport. Characteristics of the sodium-dependent system in the basallateral membrane. J Biol Chem 259, 14508-14514.

Lash, L. H., Putt, D. A., Xu, F. et al. (2007). Role of rat organic anion transporter 3 (oat3) in the renal basolateral transport of glutathione. Chem Biol Interact 170, 124-134. http://dx.doi. org/10.1016/j.cbi.2007.07.004

Lash, L. H. (2010). Renal membrane transport of glutathione in toxicology and disease. Vet Pathol 48, 408-419. http://dx.doi. org/10.1177/0300985810375811

Li, A.P. (2004). Accurate prediction of human drug toxicity: a major challenge in drug development. Chem Biol Interact 150, 3-7. http://dx.doi.org/10.1016/j.cbi.2004.09.008

Li, W., Lam, M., Choy, D. et al. (2006). Human primary renal cells as a model for toxicity assessment of chemo-therapeutic drugs. Toxicol In Vitro 20, 669-676. http://www.ncbi.nlm.nih. gov/pubmed/16289493

Lieberman, M. W., Wiseman, A. L., Shi, Z. Z. et al. (1996). Growth retardation and cysteine deficiency in gamma-glutamyl transpeptidase-deficient mice. Proc Natl Acad Sci U S A 93, 7923-7926. http://dx.doi.org/10.1073/pnas.93.15.7923

Loghman-Adham, M., Kiu Weber, C. I., Ciorciaro, C. et al. (2012). Detection and management of nephrotoxicity during drug development. Expert Opin Drug Saf 11, 581-596. http://dx.doi.org/ 10.1517/14740338.2012.691964

Meister, A., Tate, S. S. and Griffith, O. W. (1981). Gammaglutamyl transpeptidase. Methods Enzymol 77, 237-253. http:// dx.doi.org/10.1016/S0076-6879(81)77032-0

Mosmann, T. (1983). Rapid colorimetric assay for cellular growth and survival:Application to proliferation and cytotoxicity assays. J Immunol Methods 65, 55-63. http://dx.doi.org/10.1016/00221759(83)90303-4

Paolicchi,A., Sotiropuolou, M., Perego, P. et al. (2003). $\gamma$-Glutamyl transpeptidase catalyses the extracellular detoxification of cisplatin in a human cell line derived from the proximal convoluted tubule of the kidney. Eur J Cancer 39, 996-1003. http://dx.doi. org/10.1016/S0959-8049(03)00067-4

Parrinello, S., Samper, E., Krtolica, A. et al. (2003). Oxygen sensitivity severely limits the replicative lifespan of murine fibroblasts. Nat Cell Biol 5, 741-747. http://dx.doi.org/10.1038/ ncb1024

Pompella, A., De Tata, V., Paolicchi, A. et al. (2006). Expression of gamma-glutamyltransferase in cancer cells and its significance in drug resistance. Biochem Pharmacol 71, 231-238. http:// dx.doi.org/10.1016/j.bcp.2005.10.005

Rush, G. F., Smith, J. H., Newton, J. F. et al. (1984). Chemically induced nephrotoxicity: role of metabolic activation. Crit Rev Toxicol 13, 99-160. http://dx.doi.org/10.3109/10408448409034079

Russell, W. M. S. and Burch, R. L. (1959). The Principles of Humane Experimental Technique. London, UK: Methuen \& Co. Ltd.
Ryan, M. J., Johnson, G., Kirk, J. et al. (1994). HK-2: an immortalized proximal tubule epithelial cell line from normal adult human kidney. Kidney Int 45, 48-57. http://dx.doi.org/10.1038/ki.1994.6

Smith, P. L., Buffington, D. A. and Humes, H. D. (2006). Kidney epithelial cells. Methods Enzymol 419, 194-207. http://dx.doi. org/10.1016/S0076-6879(06)19009-6

Stacey, G. N. and Hartung, T. (2006). Availability, Standardization and Safety of Human Cells and Tissues for Drug Screening and Testing. In U. Marx and V. Sandig (eds.), Drug Testing In Vitro: Breakthroughs and Trends in Cell Culture Technology. Weinheim, Germany: Wiley-VCH Verlag GmbH \& Co. KGaA.

Townsend, D. M. (2003). Metabolism of cisplatin to a nephrotoxin in proximal tubule cells. J Am Soc Nephrol 14, 1-10. http:// dx.doi.org/10.1097/01.ASN.0000042803.28024.92

Voglauer, R., Grillari, J., Fortschegger, K. et al. (2005). Establishment of human fibroma cell lines from a MEN1 patient by introduction of either hTERT or SV40 early region. Int J Oncol 26, 961-970.

Wieser, M., Stadler, G., Jennings, P. et al. (2008). hTERT alone immortalizes epithelial cells of renal proximal tubules without changing their functional characteristics. Am J Physiol Renal Physiol 295, F1365-1375. http://dx.doi.org/10.1152/ajprenal. 90405.2008

Wieser, M., Limonciel, A., Pontiller, J. et al. (2014). No Title. To be submitted.

Wilmer, M. J., a Saleem, M., Masereeuw, R., et al. (2010). Novel conditionally immortalized human proximal tubule cell line expressing functional influx and efflux transporters. Cell Tissue Res 339, 449-457. http://dx.doi.org/10.1007/s00441-009-0882-y

Zhang, L. and Hanigan, M. H. (2003). Role of cysteine S-conjugate $\beta$-Lyase in the metabolism of cisplatin. J Pharmacol Exp Ther 306, 988-994. http://dx.doi.org/10.1124/jpet.103.052225

\section{Acknowledgements}

This work was supported by the Federal Ministry of Economy, Family and Youth (BMWFJ), the Federal Ministry of Traffic, Innovation and Technology (bmvit), the Styrian Business Promotion Agency SFG, the Standortagentur Tirol and ZIT - Technology Agency of the City of Vienna through the COMET-Funding Program managed by the Austrian Research Promotion Agency FFG. Additionally, RG was supported by FWF and Herzfeldersche Familienstiftung.

\section{Correspondence to}

Assoc. Prof. Dr Regina Grillari

Department of Biotechnology

University of Natural Resources and Life Sciences Vienna

Muthgasse 18

1190 Vienna

Austria

Phone: +431476546806

e-mail: regina.grillari@boku.ac.at 